\title{
A theoretical protocol of all-night EEG in polysomnography (PSG) as a preparation and precaution for repetitive transcranial magnetic stimulation (rTMS) in the elderly subjects aged 65 years and over
}

\author{
Bing H. Tang \\ Research Consultant Emeritus, New York College of Traditional Chinese Medicine, Mineola, NY, USA
}

\begin{abstract}
Objectives: A brief introduction focusing on slow wave sleep (SWS), slow wave activity (SWA), and others was presented. A hypothesis was set then proved: there were 48 among total 117 subjects, aged at least 65 years, had apnea-hypopnea index (AHI) no more than ten. Among those 48 , the presence or absence of SWS had made no difference in 12 sleep parameters. The latter were gender, age, BMI, neck circumference, stage 1 sleep duration in minutes, stage 1 sleep percentage, total sleep time, rapid eye movement in minutes, rapid eye movement percentage, sleep latency, snoring, and AHI. A protocol was then reported. It was with all-night EEG/polysomnography (PSG) in an effort as a supplementary preparation of next-day rTMS in the elderly subjects/patients at least 65 years of age. Methods: A design of retrospective sequential collection of clinical data was used.
\end{abstract}

Results: The data in this study, hence, were able to reach a protocol.

Conclusion: This study provides reasonable baseline information of an all-night EEG/PSG prior to a next-day rTMS for the elder subject aged 65 or over.

\begin{abstract}
Abbreviations: AHI: Apnea-hypopnea Index; BMI: Body Mass Index; EEG: Electroencephalography; NC: Neck Circumference; PSG: Polysomonography; rTMS: Repetitive Transcranial Magnetic Stimulation
\end{abstract}

\section{Introduction}

The analysis of rTMS progression is splintered into two portions: rTMS has materialized as both a potential treatment modality and an investigative instrument; in addition, rTMS appears to hold a significant promise for contributing to the understanding of the basic cerebral physiology over and above the pathophysiology of neuropsychiatric disorders [1-6].

Since the last few years, rTMS has emerged as a possible therapeutic modality in several neuro-psychiatric disorders [7], especially in affective disorders. The use of rTMS in therapeutic aspects has relied on the repetitive application of pulses commonly at a frequency of $1 \mathrm{~Hz}$ or more. The most part of rTMS studies in depressive patients applied higher frequency $(10-20 \mathrm{~Hz})$ rTMS over the left dorsolateral prefrontal cortex [8]. In light of the aforementioned, it appears to still be problematic even for clinical healthy subjects, especially for those elderly aged 65 years and over [9-11].

True or not, it is hard to argue for any general agreement on the therapeutic efficacy of rTMS, despite the multiple stimulation protocols [12].

One of the issues appears to be that the studied subjects'/patients' Neck Circumference (NC) and BMI (Body Mass Index) must be evaluated beforehand [13]. The regional higher-than-expected prevalence of Sleep Apnea Syndrome must be considered before any rTMS session [14].

The permanent residual effect of rTMS on EEG-power-spectra requires pre-rtms EEG power spectra as the basic guideline information. Hence, measurements of an overnight baseline activity are of relevance. The scientific base for it is that there are extremely interesting studies from all-night EEG measurements after rTMS. Those studies found cerebral local slow wave increase after rTMS during the day, suggesting rTMS induced local learning effects overnight $[15,16]$.

Interestingly, there is a TMS-based study suggests that untreated severe OSAS patients have imbalanced cortical excitabilities that enhance inhibition or decrease brain excitability when awake during the day [17].

EEG has been a well-known and commonly used clinical technique for the visualization of cerebral activity. About using EEG to explore how rTMS produces its effect on human behavior, there is a comparison between the effect of rTMS and that of 10-Hz luminance flicker [18].

The newer visualization for time-varying multichannel EEG data

Correspondence to: Bing H. Tang, Research Consultant Emeritus, New York College of Traditional Chinese Medicine, Mineola, NY, USA, E-mail: prof.bing@gmail.com

Key words: slow wave sleep (SWS), excitability, obstructive sleep apnea (OSA), polysomnography (PSG), repetitive transcranial magnetic stimulation (rTMS)

Received: July 08, 2015; Accepted: August 06, 2015; Published: August 11, 2015 
Tang BH (2015) A theoretical protocol of all-night EEG in polysomnography (PSG) as a preparation and precaution for repetitive transcranial magnetic stimulation (rTMS) in the elderly subjects aged 65 years and over

referred to as tiled parallel coordinates, is grounded on the latter method and additionally uses a two dimensional tiled layout. Nevertheless, some aspects of both methods have already been employed different ways to visualize EEG data properties [19].

There is a source analysis toolbox geared towards cognitive neuroscience researchers using MEG and EEG, called NUTMEG, including intracranial recordings. Evoked and un-averaged data can be imported to the toolbox for source analysis in either the time or timefrequency domains [20].

About the first direct electrophysiological evidence of an interaction of trans-craneal direct current stimulation, it has recently been reported [21].

It appears to be merely in two clinical studies wherein applicable fast rTMS sets $(20 \mathrm{~Hz}$ and $10 \mathrm{~Hz}$ respectively) have been in a single stimulation procedure to clinically healthy subjects, with the effects on EEG measures researched and then reported. Note that neither study revealed any fair quantity of remaining longer-lasting effects on EEG power spectra $[22,23]$.

Through non-rapid-eye-movement (NREM) sleep an EEG is predominated by slow waves of high amplitude, which are produced by mountains of neurons swapping from a depolarized up-condition to a hyperpolarized down-condition [22,23].

In many different species, sleep slow waves is commonly designated by way of slow wave activity (SWA); the EEG power is from the range of from one to $4.5 \mathrm{~Hz}$. SWA usually is in a homeostatic condition [24,25]. Explicitly, SWA upsurges with the period of previous wakefulness, which is waning exponentially through sleep as well. Hence, SWA offers a consistent gauge of sleep pressure and might be related to the revitalizing drive of sleep [26,27]. In a study with high-density-EEG in humans, it has been noted that sleep SWA is regionally increased status post a cerebral visuomotor learning process. Nevertheless, such a phenomenon does not happen after a cerebral function that does not require learning [28].

An important advance has come from recent works showing that sleep SWA can be regulated locally in the cerebral cortex. Some of these works might lead to a connection between cerebral regional SWA regulation and synaptic plasticity [28]. Hence, in a study, sleep SWA was noted nocturnally to be locally decreased over human's right cerebral sensorimotor cortex of a right-handed subject, only if a subject's left arm had been immobilized during the day, leading to a deterioration in motor performance and to a decreased somatosensory and motor evoked potentials (www.The freelibrary. 2012) [29,30]. These experiments hint that sleep SWA is more or less influenced by plastic changes in cerebral local cortical connections, particularly, that SWA should enhance with synaptic potentiation and lessen with synaptic repression [31,32]. Knowing that there is a relationship between sleep function and synaptic homeostas is (Tononi et al 2006) [32], the potential theory appears to be reinforced by computer simulations displaying that solid synapses lead to increased SWA by neuronal synchronization, while pathetic synapses have the contrary outcome [31].

There is a potential for producing an antidepressant response with rTMS without eliciting seizure activity, eliciting focal seizures with rTMS, and the possibility of using rTMS to focally modulate seizure induction as well as spread with ECT to optimize treatment [31].

When delivered during the performance of a task, rTMS affects behavior by producing transient "virtual lesions" in targeted brain tissues. Nevertheless, findings of rTMS-related improvements in performance are not easy to settle with the aforementioned assumption. Some study suggests that rTMS affects performance by biasing endogenous task-related oscillatory dynamics, rather than creating a "virtual lesion" [33].

It appears important to speculate that for elderly people, even for the healthy individuals, PLMS is as a function of age [34]. PLMS (Periodic Limb Movement Syndrome) has profound relationship with sleep microstructure; their relationship with the macrostructural parameters are less well established but definitely the latter cannot be excluded. Noted that sleep disorder manifestation varies by patients; it is also noted that Rest Leg Syndrome (RLS) and PLMS are both very dependent on ferritin and its related substance [35].

On using EEG to explore how rTMS produces its effect on human behavior, there has been some comparison for the effects of rTMS on ongoing brain activity and behavior to the effects of $10-\mathrm{Hz}$ luminance flicker [18].

There has been a study of total 192 patients were randomly allocated to received rTMS treatment; such a study well demonstrated patients' safety and tolerability for rTMS [36].

There has been an article presenting that the elevated AHI was largely due to central apnea, but not obstructive apnea, for the age range from 65 years and over [37], hence, central apnea predicts mortality above age 65 years; others also published similar data [38].

\section{Subjects and methods}

Changhua Christian Hospital Medical Center's Internal Research Board and Ethical Committee approved this study, which was in conformity with the Declaration of Helsinki. Consents for both personal and medical data were obtained. At the time of their initial evaluation and enrollment, they all authorized clinical data in the research database to be used. Each was given a written informed consent.

In this study, a design of retrospective sequential collection of clinical data was used to explore community dwelling older adults in permitting the use of their all-night EEG data for clinical research.

During the period from 1 January 2002 to 31 January 2003, the Sleep Medicine Center of Changhua Christian Hospital Medical Center, Taiwan admitted no patients with chronic obstructive lung disease, cardiac failure, epilepsy or any other neurologic or psychiatric diseases; as there was no physician at night in Sleep Medicine Center. Among the consecutive subjects who underwent all-night PSG during that period, there were all together 1,087 cases. Those data that belong to individuals who were younger than 65 years were excluded. Hence the inclusion criteria were: the chief complaint of sleep disturbance and aged 65 years and over.

From total 1,087 cases, there were 124 participants aged from 65 to 88.5 years, no one was demented or sleep deprived. No pair of individuals was related to each other. Among them, there were 117 that had both NC and BMI data available. There were 11 octogenarians among the total 124. Consents for both personal and medical data were obtained. At the time of their initial evaluation and enrollment, they all authorized clinical data in the research database to be used. Each was given a written informed consent.

All clinical data used were in accordance with the same spirit of 
Tang BH (2015) A theoretical protocol of all-night EEG in polysomnography (PSG) as a preparation and precaution for repetitive transcranial magnetic stimulation (rTMS) in the elderly subjects aged 65 years and over

Health Insurance Portability and Accountability Act regulations (HIPAA) (U S Health Insurance 1996) [39]. Subjects completed the Epworth Sleepiness Scale and Quality of Life SF-36 (QOL) sleep questionnaires. They were ambulatory. The results of PSG were analyzed on the basis of AHI. The apneic subjects showed a reduction in QOL, which was proportional to severity in apnea. There was a significant decrease in all domains (physical functioning, role physical problems, bodily pain, general health perceptions, vitality, social functioning and emotional problems) for apneic with AHI $>30$, who generally were sleepy and did not participate in physical activities $(\mathrm{P}<0.05)$.

The sleep recordings were scored using Rechtschaffen and Kales criteria [40]. An apneic event was defined as a decrease in airflow of more than $90 \%$ for a minimum of 10 seconds. A hypopneic event was scored if airflow decreased more than 30\%, and desaturations required a $3 \%$ reduction in oxygen saturation for a minimum of ten seconds. Subject with an AHI of 5 or more was considered as having a diagnosable case of obstructive sleep apnea (OSA). Apnea was defined as a decrease in airflow of $\geq 90 \%$ for a minimum of 10 seconds. Hypopnea was defined as $\geq 30 \%$ decrease in airflow and desaturations required $a \geq 3 \%$ decrease in oxygen saturation for a minimum of 10 seconds. PSG was conducted using a full 18-channel montage. A certified PSG technician conducted the PSG study, although subjects determined their own bedtime for sleep at night. A digital PSG system (Alice 4, Respironic, US), and a finger pulse oxymetry (model N 200, Nellcor, Hayward, Calif.) were used. Its Recordings included recorded central and occipital electroencephalogram (EEG) derivations (C3, C4, $\mathrm{O} 1, \mathrm{O} 2$ ), bilateral electrooculogram (left outer canthus and right outer canthus), submental and anterior tibialis electromyogram (EMG), electrocardiogram, nasal/oral airflow using a thermistor, respiratory effort using chest and abdominal inductance belts.

The diagnosis of Sleep-disordered Breathing (SDB) has been defined when the subject has AHI score of as $\geq 5$.

\section{Statistics}

Matlab $^{\oplus}$ version 2010b was used. A normality of distribution of the data has been tested by using Kolmogrove-Smirnov's method. The Variances of SWS and the other clinical attributes such as AHI, BMI, stage 1 sleep duration in minutes, neck circumference and so on were presented to ascertain that their variances differed from each other.

Wilcoxon rank sum test (WRST) was then used. Independent t-test was used for the aim of contrast. The $p$ value significant level of 0.05 was set. Whenever it was feasible, a different $p$ value was set for its correction.

\section{Results}

\section{Gender differences}

The gender difference included mean height, weight and cervical circumference. They were less in females. Female subjects had mean BMI of $26.550 \mathrm{~kg} / \mathrm{m}^{2}$, and males 25.19. The mean BMI of total 117 subjects was $25.574+-4.521 \mathrm{kgs} / \mathrm{m}^{2}$, while that of control was 23.768 $+-3.662 \mathrm{~kg} / \mathrm{m}^{2}$, the difference was significant (the $\mathrm{p}$-value $<0.0009$ ). Pearson's correlation coefficient between height and BMI was significantly different between males $(-0.227)$ and females $(-0.0854)$. The female subjects were about one year older in average than the male.

\section{The distribution pattern of all the clinical attributes (variables)}

Kolmogorov-Smirnov (KS) test was performed on all 12 attributes and for the two groups (SWS present and SWS absent) respectively (Table 1). When SWS was present in the all-night EEG, the p-values of Sleep latency, Sleep efficiency in \%, and Snoring are all below 0.05, therefore, the null hypothesis of KS test was rejected. The distribution was not normal. When SWS was absent, the p-values of Sleep latency was the one attribute with the p-value $<0.05$, which was the only p-value less than the significant level of 0.05 , the null hypothesis of KS test was rejected. The distribution of Sleep latency thus was not normal for those without SWS.

The variance of these 12 clinical attributes was then measured to ascertain and confirmed that they were different from each other.

The variance of 12 clinical attributes (Table 2). The mean and standard deviation of the 12 attributes are presented in Table 3. All distribution of attributes was in normal distribution (Table 3). A twosample hypothesis test was done for the clinical attributes, Wilcoxon Rank Sum test was used, and followed with a t- test for comparison (Table 4).

For all the four different degrees of severity of SDB in this study, Wilcoxon Rank Sum test was used to evaluate Group-wise two-sample hypothesis.

With 81 subjects SWS-present versus 36 subjects SWS-absent, the result of Wilcoxon Rank Sum test reveals that a p-value was 0.04567 , which was $<0.5$. As what could be visualized in Table 5 , there were 20 subjects in their Zero degree of severity of SRB. Among those 20, there

Table 1. Kolmogorov-Smirnov test result.

\begin{tabular}{|l|c|c|c|c|c|c|c|c|c|c|c|c|c|c|c|c|c|c|}
\hline & Age & BMI & NC(cm) & Latency & Stage 1 & Stage 1\% & TST & Eff\% & REMmin & REM\% & Snore & AHI & & & & & & \\
\hline SWS present & 0.5452 & 0.8260 & 0.3860 & 0.0000 & 0.0784 & 0.1061 & 0.1581 & 0.0376 & 0.9902 & 0.9854 & 0.0012 & 0.0717 \\
\hline SWS absent & 0.7438 & 0.4163 & 0.7475 & 0.0023 & 0.9866 & 0.6073 & 0.6015 & 0.2955 & 0.9115 & 0.6327 & 0.6852 & 0.2840 \\
\hline
\end{tabular}

All distribution of attributes was in normal distribution

Table 2. Variances of 12 Clinical Attributes.

\begin{tabular}{|c|c|c|c|c|c|c|c|c|c|c|c|c|c|}
\hline & \# of cases & Age & BMI & $\mathrm{NC}(\mathrm{cm})$ & Latency & Stage 1 & Stage 1\% & TST & Eff $\%$ & REMmin & REM\% & Snore & AHI \\
\hline SWS present & 81 & 18.47 & 19.21 & 9.05 & 1536.41 & 3822.12 & 180.98 & 9222.08 & 347.04 & 623.52 & 32.43 & a1002649.77 & 519.48 \\
\hline SWS absent & 36 & 30.46 & 23.04 & 13.37 & 1998.90 & 1564.56 & 86.57 & 6413.27 & 253.69 & 852.77 & 44.99 & 409188.18 & 337.04 \\
\hline
\end{tabular}

Since the normality has been checked, the variance of these 12 clinical attributes was then measured in order to ascertain that they were different from each other, and finally confirmed to be so. 
Tang BH (2015) A theoretical protocol of all-night EEG in polysomnography (PSG) as a preparation and precaution for repetitive transcranial magnetic stimulation (rTMS) in the elderly subjects aged 65 years and over

Table 3. The mean and standard deviation of the 12 attributes according to the presence vs. absence of SWS.

\begin{tabular}{|c|c|c|c|c|}
\hline \multirow{2}{*}{\begin{tabular}{|l|} 
Slow Wave Sleep \\
Numbers of Cases \\
\end{tabular}} & \multicolumn{2}{|c|}{ SWS present } & \multicolumn{2}{|c|}{ SWS absent } \\
\hline & 81 & & 36 & \\
\hline Statistics & Mean & Std Dev & Mean & Std Dev \\
\hline Age & 71.35 & 4.30 & 72.25 & 5.52 \\
\hline BMI & 25.31 & 4.38 & 26.39 & 4.80 \\
\hline $\mathrm{NC}(\mathrm{cm})$ & 37.15 & 3.01 & 35.93 & 3.66 \\
\hline Sleep Latency in mins & 24.82 & 39.20 & 23.53 & 44.71 \\
\hline Stage I Sleep duration in mins & 97.73 & 61.82 & 76.69 & 39.55 \\
\hline Stage I Sleep in \% within Total Sleep time & 22.84 & 13.45 & 18.62 & 9.30 \\
\hline TST (Total Sleep time) & 328.09 & 96.03 & 321.35 & 80.08 \\
\hline Sleep Effciency in \% & 73.01 & 18.63 & 73.09 & 15.93 \\
\hline REM Sleep in mins & 43.85 & 24.97 & 44.90 & 29.20 \\
\hline REM Sleep in \% & 10.24 & 5.70 & 10.80 & 6.71 \\
\hline Snoring & 809.85 & 1001.32 & 767.86 & 639.68 \\
\hline AHI & 28.15 & 22.79 & 19.60 & 18.36 \\
\hline
\end{tabular}

Table 4. Group-wise two-sample hypothesis test results (using Wilcoxon Rank Sum Test).

\begin{tabular}{|l|c|c|c|c|c|c|c|c|c|c|c|c|c|c|c|c|c|c|}
\hline \# of cases & Age & BMI & NC(cm) & Latency & Stage 1 & Stage 1\% & TST & Eff\% & REMmin & REM\% & Snore & AHI \\
\hline Wilcoxon rank sum text & 0.4877 & 0.4392 & 0.0845 & 0.4496 & 0.1239 & 0.1661 & 0.6157 & 0.8018 & 0.7565 & 0.4803 & 0.3194 & 0.0456 \\
\hline t-test & 0.3067 & 0.2624 & 0.0500 & 0.8775 & 0.0625 & 0.0871 & 0.8376 & 0.8494 & 0.7648 & 0.5735 & 0.8580 & 0.0380 \\
\hline
\end{tabular}

For the clinical attributes, Wilcoxon Rank Sum test was first used, and was then followed with a t- test for the aim of comparing the test outcome.

Table 5. According to the Degree of Severity (an elevated AHI) of SDB in this study.

\begin{tabular}{|l|c|c|c|c|}
\hline Degree of Severity & 0 & 1 & 2 & 3 \\
\hline \# of Cases & 20 & 28 & 27 & 42 \\
\hline Age & 0.4033 & 0.3134 & 0.5747 & 0.4326 \\
\hline BMI & 0.2875 & 0.7502 & 0.0137 & 0.2555 \\
\hline NC $(\mathrm{cm})$ & 0.0215 & 0.7695 & 0.3520 & 0.2350 \\
\hline Sleep Latency in mins & 0.7040 & 0.9577 & 0.1301 & 0.8225 \\
\hline Stage I Sleep duration in mins & 0.7611 & 0.0438 & 0.9805 & 0.7126 \\
\hline Stage I Sleep in \% & 1.0000 & 0.0894 & 0.8836 & 0.8601 \\
\hline TST & 0.3233 & 0.7103 & 0.6255 & 0.6423 \\
\hline Efficient of Sleep in \% & 1.0000 & 0.9788 & 0.8644 & 0.7731 \\
\hline REM Sleep in mins & 0.4249 & 0.3007 & 0.1641 & 0.4908 \\
\hline REM Sleep in \% & 0.4941 & 0.2223 & 0.0637 & 0.6537 \\
\hline Snoring & 0.4925 & 0.1518 & 0.0509 & 0.7609 \\
\hline AHI & 0.8492 & 0.0466 & 0.9805 & 0.3960 \\
\hline
\end{tabular}

were some having SWS vs. others without SWS, the Wilcoxon Rank Sum test result reveals that for the only one among all the 12 variables, the variable of NC's p-value was 0.0215 , which was $<0.05$ at an initial glance; nevertheless, if one considers correction of the possibility of false positive results due to the repetition of the statistical univariate analyses, then it would be indicated for using Bonferroni correction to divide 0.05 by 12 and then obtained the corrected threshold. In that case one would have 0.004 . This means that the actual end result was not statistically different if one had corrected for the repetition of tests.

Those 28 subjects of First degree severity (AHI ranges between 5 and 10), among them, subjects with SWS versus subjects without, the Wilcoxon Rank Sum test result revealed for Stage 1 sleep by minutes, its $\mathrm{p}$ value was 0.0438 , after correction then the threshold was 0.004 . This means that the result was not statistically different when one had corrected for the repetition of tests.

About the 27 subjects of Second degree severity, among them, subjects with SWS vs. subjects without, Wilcoxon Rank Sum test result revealed that for BMI, its p value was 0.0137 , it looked like significant, but after correction, it was larger than the actual threshold of 0.004 , hence, the end result was not statistically different either (Table 5).

AHI had already been $>15$, there was little value to be worrisome in this study. With 42 subjects of Third degree severity, among them, subjects with SWS vs. subjects without, the Wilcoxon Rank Sum test result revealed that there was no significant p-value found; this is an very important precaution for the clinician plans to do rTMS on patients.

\section{Discussion}

Generally, the dominant frequency in the scalp EEG of human adults is in the alpha frequency range $(8-12 \mathrm{~Hz})$ and can be detected as prominent peak in the frequency spectrum. This study proves the hypothesis that when all subjects' AHI not $>10$, there was no difference in the 12 sleep parameters regardless of the presence/absence of SWS.

Statistically, if one has a Gaussian distribution of a variable (e.g. the magnitude of SWS) and divides the population in two groups based on a median of that variable, it is expected that the means of the variable in the two groups are different. In this study, the two groups of SWS+ and SWS-, were not divided by the median of SWS.

About the scalp recordings of EEG, they have been employed in association with rTMS. These two tests are safety measures in monitoring ongoing EEG activity and both are neurophysiologic tools in examining the specific effects induced by the magnetic stimulus on the EEG changing. Thus far, the pathophysiological and therapeutic mechanisms of rTMS have not yet been fully clarified.

Note that the 12 sleep parameters here are those already been used in this study; additionally, There are certain other parameters that can still be added to the 12 sleep parameters here such as each sleep stage percentage and the arousal index. 
Tang BH (2015) A theoretical protocol of all-night EEG in polysomnography (PSG) as a preparation and precaution for repetitive transcranial magnetic stimulation (rTMS) in the elderly subjects aged 65 years and over

Without rTMS data in these subjects, it appears that any conclusion regarding possible safety hazards might be precluded, though theoretically it seems reasonable.

Why this study has not accepted the usual sleeping environment at home but carried out all the measurement in a sleep medicine laboratory? Does not moving to the sleep laboratory worsen sleep? It has been determined that most people adapt quickly to the laboratory sleep environment and can fall asleep with usual ease (or show a characteristic magnitude of difficulty) after a short period of reworking. These remarks trigger the common protocol used in sleep studies, which is to carry out multiple nights of PSG sleep evaluation and to discern the first night as an adaptation night from the rest of complete analysis.

Trying to reduce as much as possible the effect of a strange sleeping environment has resulted to technologies developed for home monitoring; nevertheless, monitoring at home does have its own other technical problems, especially for the elderly. It appears that we all should take precaution whenever we are contemplating rTMS for an elderly.

After going through literature reviewing, it appears that there is a failure to locate any references good enough to clearly explain the rationale for the need to record sleep PSG before rTMS sessions in order to substantiate the claim such as what has been cited in this study, even if not directly assessing this aspect, but at least is indicating the need to some extent.

Why a PSG in the evening-night before rTMS might be a safety hazard? It appears that, thus far, nobody knows if the finding of a presence of overnight sleep apnea is a safety hazard for the next day's rTMS. The real key point will be: does the presence of sleep apnea modify the brain structure excitability? The answer seems to be YES. Next, is the alteration in neuronal excitability correlated with the severity of sleep apnea? In other words, does the alteration in neuronal excitability correlated with the elevated AHI)? Third, do these alterations require an adjustment from rTMS?

Last year, there was a TMS-based study suggested that untreated severe OSAS patients had imbalanced cortical excitabilities that enhanced inhibition or decreased brain excitability when awake during the day [26].

Another important question, hence, remained to be answered is as following: What are indications for rTMS in patients with sleep apnea? In other words, is rTMS indicated in patients with sleep apnea?

The aforesaid are the questions to be answered by all. As having been well known that sleep deprivation has an influence on neuronal excitability as does sleep apnea, that is, as well, the reason why in this study, sleep deprivation have been excluded as the first possibility in the flow chart here (Figure 1).

True or not, it is hard to determine whether or not the severity of sleep apnea correlates with sleep deprivation. Thus far, there is no proven evidence of a therapeutic potential of rTMS for sleep apnea.

Nevertheless, most top level EEG laboratories use scales of quality of sleep and have an interview about psychoactive substances use and/or abuse, as well, asking about the quality of sleep the night before an EEG/ PSG recording. If the quality of the prior EEG/PSG is not satisfactory, then the next daytime EEG/rTMS has to be postponed. This means that there is a consensus on the fact that a suboptimal quality of sleep the day before the daytime EEG/rTMS recording prevents a reliable future

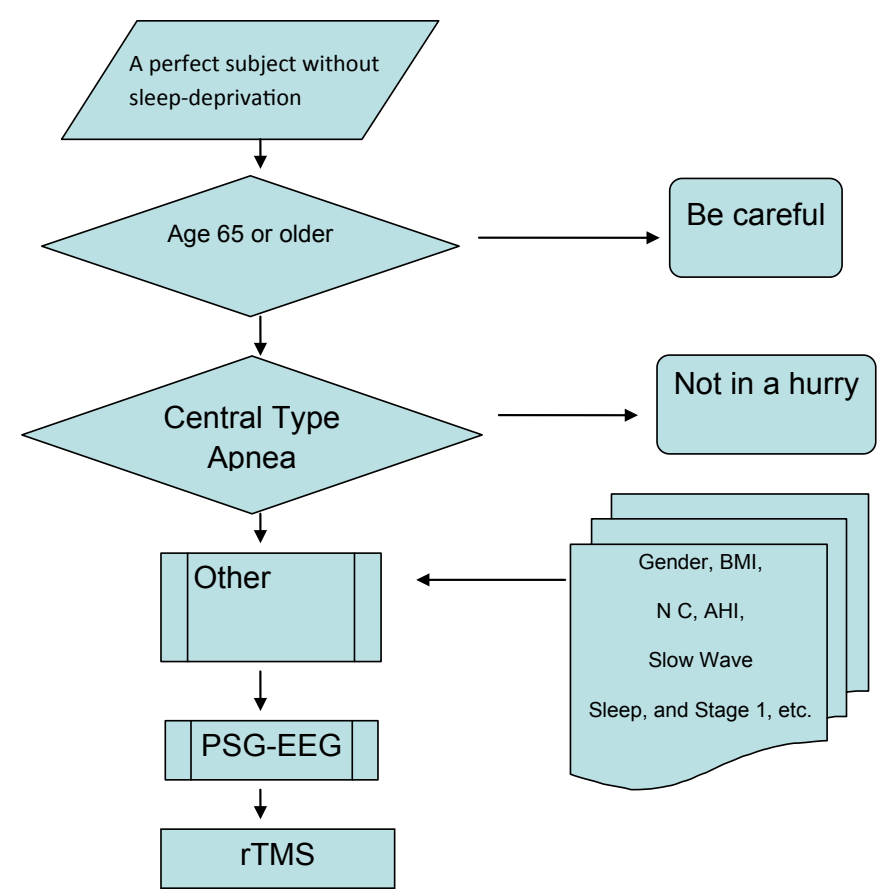

Figure 1. Flow Chart of the all-night EEG/PSG for rTMS.

According to the direction of arrows, from the top downward, first thing to do is to ascertain that the studied subject is not sleep deprived. BMI, N C, and AHI are needed to be evaluated in addition to the sleep parameters.

EEG/rTMS recording. Under such a circumstance, there are several not mutually exclusive actions that one can readily take in order to reduce the trepidation of the PSG procedures, which might take place. For example:

Employ a familiar PSG recording in the day 1, perform an experimental PSG recording in the day 2, and then record the wake resting and event-related EEG/TMS recordings in the day 3 . Or use an actigraphy and a self-report scale, or a reliable reporter to judge the quality of the sleep for more than 3 days but less than 7 days before the wake resting and event-related EEG/rTMS recordings.

How to ascertain that the PSG does induce changes in the awake resting and event-related EEG/TMS recordings? One has to strictly follow the golden rules for any clinical intervention trials of phase II. One, as well, has to delineate the sample size on the basis of the estimation of the effect size of the dependent (primary end point) variable. One ought to design a randomized, placebo controlled, crossover trial. Following it, one should test the null hypothesis that the primary endpoint is not going to be altered by PSG, which has been done the night before a daytime EEG/rTMS recording.

The weakness of this article appears to be lack of a contribution to a relationship between sleep EEG structure and daytime cortical excitability as revealed by rTMS. The possible feebleness of this study appears to be on the fact of its privation of rTMS' effect of strengths, frequencies, and durations of stimulation on the EEG power spectra over a specific cerebral area stimulated with rTMS.

\section{Conclusion}

The result from this study provides direct evidence showing no difference between SWS present and SWS absent two different elder groups when their AHI is not greater than ten. 
Tang BH (2015) A theoretical protocol of all-night EEG in polysomnography (PSG) as a preparation and precaution for repetitive transcranial magnetic stimulation (rTMS) in the elderly subjects aged 65 years and over

Hence, for subject/patient aged 65 years and over, it appears to be convinced that a prior in-laboratory all-night PSG is helpful in order to exclude the very frequent disorders that can be found, but are not always noticeable or reported by the subjects whoever in this age group having sleep apnea, periodic limb movements, chronic tinnitus and so on.

The first night-effect of the EEG/PSG recording procedure most likely will be moderate and does not really represent a significant sleep deprivation induced by PSG. Sleep EEG should always be recorded during PSG in order to correspond between EEG and other sleep events.

For a pilot study, instead of considering about PSG via a full 18-channel montage, it is suggestive to include a relatively smaller number of EEG channels, such as (F3-A2, C3-A3, O1-A2, F4-A1, C4$\mathrm{A} 1, \mathrm{O} 2-\mathrm{A} 1)$.

\section{References}

1. Boutros NN, Berman RM, Hoffman R, Miano AP, Campbell D, et al. (2000) Electroencephalogram and repetitive transcranial magnetic stimulation. Depress Anxiety 12: 166-169. [Crossref]

2. Pascual-Leone A, Davey N, Rothwell J, Wassermann EM, Puri BK (2002) Handbook of Transcranial Magnetic Stimulation, Hodder Arnold.

3. Fitzgerald PB, Fountain S, Daskalakis ZJ (2006) A comprehensive review of the effects of rTMS on motor cortical excitability and inhibition. Clin Neurophysiol 117: 25842596. [Crossref]

4. Rossi S, Hallett M, Rossini PM, Pascual-Leone A; Safety of TMS Consensus Group (2009) The Safety of TMS Consensus Group, Safety, ethical considerations, and application guidelines for the use of transcranial magnetic stimulation in clinical practice and research. Clin Neurophysiol 120: 2008-2039. [Crossref]

5. Transcranial Magnetic Stimulation (TMS) National Alliance on Mental Illness. 2012.

6. http://www.nami.org/Content/ContentGroups/Helpline1/Transcranial_Magnetic_ Stimulation (rTMS).htm. [Retrieved 11, 15, 2012]

7. Dimyan MA, Cohen LG (2010) Contribution of transcranial magnetic stimulation to the understanding of functional recovery mechanisms after stroke. Neurorehabil Neural Repair 24: 125-135. [Crossref]

8. Feredoes E, Tononi G, Postle BR (2006) Direct evidence for a prefrontal contribution to the control of proactive interference in verbal working memory. Proc Natl Acad Sci US A 103: 19530-19534. [Crossref]

9. Ferrarelli F, Massimini M, Peterson MJ, Riedner BA, Lazar M, et al. (2008) Reduced evoked gamma oscillations in the frontal cortex in schizophrenia patients: a TMS/EEG study. Am J Psychiatry 165: 996-1005. [Crossref]

10. Grosbras MH, Paus T (2003) Transcranial magnetic stimulation of the human frontal eye field facilitates visual awareness. Eur J Neurosci 18: 3121-3126. [Crossref]

11. Gazzaley A, Nobre AC (2012) Top-down modulation: bridging selective attention and working memory. Trends Cogn Sci 16: 129-135. [Crossref]

12. Prinz PN, Peskind ER, Vitaliano PP, Raskind MA, Eisdorfer C, et al. (1982) Changes in the sleep and waking EEGs of nondemented and demented elderly subjects. $\mathrm{J} \mathrm{Am}$ Geriatr Soc 30: 86-93. [Crossref]

13. Ilmoniemi RJ, Virtanen J, Ruohonen J, Karhu J, Aronen HJ, et al. (1997) Neuronal responses to magnetic stimulation reveal cortical reactivity and connectivity. Neuroreport 8: 3537-3540. [Crossref]

14. Tang B, Tiu L (2009) The Impact of Cultural Changes on the Relationship between Senior Sleep Disturbance and Body Mass Index among Older Adults in Two Asian Societies. In: Soriento YE (Ed.), Melatonin, Sleep andInsomnia. Nova Publishing, N Y. 161-190

15. Puvanendran K, Goh KL (1999) From snoring to sleep apnea in a Singapore population. Sleep Res Online 2: 11-14. [Crossref]

16. Massimini M, Ferrarelli F, Esser SK, Riedner BA, Huber R, et al. (2007) Triggering sleep slow waves by transcranial magnetic stimulation. Proc Natl Acad Sci U S A 104: 8496-8501. [Crossref]

17. Huber R, Määttä S, Esser SK, Sarasso S, Ferrarelli F, et al. (2008) Measures of cortical plasticity after transcranial paired associative stimulation predict changes in electroencephalogram slow-wave activity during subsequent sleep. $J$ Neurosci 28 : 7911-7918. [Crossref]

18. Yoo EY, Kim HJ, Lim YH, Koo DL, Hong SB (2010) Altered cortical excitability in patients with untreated obstructive sleep apnea syndrome. Sleep Med 11: 857-861. [Crossref]

19. Johnson JS, Hamidi M, Postle BR (2010) Using EEG to explore how rTMS produces its effects on behavior. Brain Topogr 22: 281-293. [Crossref]

20. Huber R, Ghilardi MF, Massimini M, Ferrarelli F, Riedner BA, et al. (2006) Arm immobilization causes cortical plastic changes and locally decreases sleep slow wave activity. Nat Neurosci 9: 1169-1176. [Crossref]

21. Dalal SS, Zumer JM, Guggisberg AG, Trumpis M, Wong DD, et al. (2011) MEG/EEG source reconstruction, statistical evaluation, and visualization with NUTMEG. Comput Intell Neurosci 2011: 758973. [Crossref]

22. Zaehle T, Rach S, Herrmann CS (2010) Transcranial alternating current stimulation enhances individual alpha activity in human EEG. PLoS One 5: e13766. [Crossref]

23. Hamidi M, Slagter HA, Tononi G, Postle BR (2009) Repetitive transcranial magnetic stimulation affects behavior by biasing endogenous cortical oscillations. Front Integr Neurosci a: 3. [Crossref]

24. Steriade M (2003) The corticothalamic system in sleep. Front Biosci 8: d878-899. [Crossref]

25. Bliss TV, Lomo T (1973) Long-lasting potentiation of synaptic transmission in the dentate area of the anaesthetized rabbit following stimulation of the perforant path. $J$ Physiol 232: 331-356. [Crossref]

26. Manconi M, Ferri R, Zucconi M, Bassetti CL, Fulda S, et al. (2012) Dissociation of periodic leg movements from arousals in restless legs syndrome. Ann Neurol 71: 834844. [Crossref]

27. Esser SK, Huber R, Massimini M, Peterson MJ, Ferrarelli F, et al. (2006) A direct demonstration of cortical LTP in humans: a combined TMS/EEG study. Brain Res Bull 69: 86-94. [Crossref]

28. Peinemann A, Reimer B, Löer C, Quartarone A, Münchau A, et al. (2004) Long-lasting increase in corticospinal excitability after 1800 pulses of subthreshold $5 \mathrm{~Hz}$ repetitive TMS to the primary motor cortex. Clin Neurophysiol 115: 1519-1526. [Crossref]

29. Quartarone A, Bagnato S, Rizzo V, Morgante F, Sant'angelo A, et al. (2005) Distinct changes in cortical and spinal excitability following high-frequency repetitive TMS to the human motor cortex. Exp Brain Res 161: 114-124. [Crossref]

30. Fusile JP, Arges GS, Barrett LB (2002) Straight talk: new approaches in health care. HIPAA: deadlines are looming. Are providers prepared? Mod Healthc 32: 37-40. [Crossref]

31. www.thefreelibrary.com/Sleep+disorder+manifestations+vary+by patients [Retrieved $11,15,2012]$

32. Hill S, Tononi G (2005) Modeling sleep and wakefulness in the thalamocortical system. J Neurophysiol 93: 1671-1698. [Crossref]

33. Tononi G, Cirelli C (2006) Sleep function and synaptic homeostasis. Sleep Med Rev 10: $49-62$.

34. Krystal AD, West M, Prado R, Greenside H, Zoldi S, et al. (2000) EEG effects of ECT implications for rTMS. Depress Anxiety 12: 157-165. [Crossref]

35. Pennestri MH, Whittom S, Adam B, Petit D, Carrier J, et al. (2006) PLMS and PLMW in healthy subjects as a function of age: prevalence and interval distribution. Sleep 29: 1183-1187. [Crossref]

36. Monconi M, Ferri R, Zucconi M, Bassetti CL, Fulda S, et al. (2005) Dissociation of periodic leg movements and wakefulness in the thalamocortical system. $J$ Neurophysiol 93:1671-1698.

37. Langguth B, Landgrebe M, Frank E, Schecklmann M, Sand PG, et al. (2014) Efficacy of different protocols of transcranial magnetic stimulation for the treatment of tinnitus: Pooled analysis of two randomized controlled studies. World J Biol Psychiatry 15 276-285. [Crossref]

38. Ancoli-Israel S, Engler RL, Friedman PJ, Klauber MR, Ross PA, et al. (1994) Comparison of patients with central sleep apnea. With and without Cheyne-Stokes respiration. Chest 106: 780-786. [Crossref]

39. Lavie L, Lavie P (2007) Elevated plasma homocysteine in older shift-workers a potential risk factor for cardiovascular morbidity. Chronobiol Int 24: 115-128. [Crossref] 
Tang BH (2015) A theoretical protocol of all-night EEG in polysomnography (PSG) as a preparation and precaution for repetitive transcranial magnetic stimulation (rTMS) in the elderly subjects aged 65 years and over

40. U S Health Insurance Portability and Accountability Act of 1996 (HIPAA) Privacy and Security Rules.
41. Rechtschaffen A, Wolpert EA, Dement WC, Mitchell SA, Fisher C (1963) Nocturnal Sleep of Narcoleptics. Electroencephalogr Clin Neurophysiol 15: 599-609. [Crossref]

Copyright: (2015 Tang BH. This is an open-access article distributed under the terms of the Creative Commons Attribution License, which permits unrestricted use, distribution, and reproduction in any medium, provided the original author and source are credited. 